\title{
Comparison of supervised and unsupervised models of physical therapy in total knee arthroplasty: systematic review
}

\begin{abstract}
Background: Total knee arthroplasty (TKA) is an effective surgical treatment for advanced osteoarthritis. TKA must always be associated with postoperative physical rehabilitation provided by a physical therapist in order to restore functionality. As TKA-related health costs increase, an efficient rehabilitation program is needed. However, the physical therapy models for TKA patients are questionable.

Objective: The objective of the systematic review is to analyze the evidences that deal with different models of physical rehabilitation and compare the effectiveness of the supervised treatment as well as the in home exercise programs without supervision.

Methods/design: The databases Pubmed, Embase, Lilacs, The Cochrane Library, and Trip were searched for randomized clinical trials in any language and publication medium. The outcomes of interest were muscle strength, physical mobility and range of motion, atrophy, joint pain, and quality of life. The Cochrane Handbook for Systematic Reviews of Interventions was used for assessing the methodological quality of the studies.
\end{abstract}

Results: The search returned 1133 studies. Of these, only five were included. Although the studies were heterogeneous, three studies investigated knee flexion range of motion, but this outcome did not differ statistically between groups after six months.

Conclusion: In conclusion, the evidence for supervised and unsupervised models of physical therapy for TKA patients is inconclusive because important outcomes have not been investigated, and the pertinent studies have low methodological quality.

Keywords: total knee arthroplasty, outpatient care, rehabilitation
Volume 2 Issue 5 - 2017

\author{
Thomas Fernando Coelho Pesavento,' 'Vania \\ Fernanda Clemente Agner, ${ }^{2}$ Eduarda Santos \\ Puga, ${ }^{3}$ Gustavo José Martiniano Porfírio, ${ }^{4}$ \\ Maria Stella Peccin ${ }^{5}$ \\ 'Physiotherapist, Master's Student in the Postgraduate Evidence- \\ Based Healthcare Program, Universidade Federal de São Paulo, \\ Brazil \\ ${ }^{2}$ Physiotherapist, Master's Student in the Postgraduate \\ Interdisciplinary Health Sciences Program, Universidade Federal \\ de São Paulo, Brazil \\ ${ }^{3}$ Librarian, Evidence-Based Healthcare Program, Universidade \\ Federal de São Paulo (UNIFESP), Brazil \\ ${ }^{4}$ Physiotherapist, Student in the Postgraduate Evidence-Based \\ Healthcare Program, Universidade Federal de São Paulo, Brazil \\ ${ }^{5}$ Physiotherapist, Titular Professor, Department Evidence-Based \\ Healthcare Program, Universidade Federal de São Paulo, Brazil
}

\begin{abstract}
Correspondence: Thomas Fernando Coelho Pesavento, Physiotherapist, Master's Student in the Postgraduate EvidenceBased Healthcare Program, Universidade Federal de São Paulo (UNIFESP), Zipcode 04039-00 I, Brazil, Tel +55 I I55752970, Email thomas-fisio@hotmail.com
\end{abstract}

Received: August 17,2017 | Published: December 27, 2017
Abbreviations: OA, osteoarthritis; TKA, total knee arthroplasty; KOA, knee osteoarthritis; WOMAC, western ontario master universities; KOOS, knee injury osteoarthritis outcome score; KSS, knee society score; OKS, oxford knee score; VAS, visual analogue scale; CPM, continuous passive motion; NMES, neuromuscular electrical stimulation; ROM, range of motion; SD, standard deviation

\section{Background}

Osteoarthritis (OA) is the most common form of arthritis, a chronic disease that affects the joints and progressively changes joint cartilage. OA has a complex pathogenesis, since the imbalance that occurs in cartilage and bone tissue leads to progressive erosion, osteophytes, bone marrow injury, and synovial membrane inflammation. ${ }^{1}$ Changes in biomechanical and biochemical patterns favor the emergence of OA. $^{2}$

The knee has the highest OA prevalence of all joints. Knee OA (KOA) affects $75 \%$ of the population aged more than 65 years, making it a public health problem. ${ }^{3}$ If not properly treated in the early stages, advanced KOA causes intense pain, functional disability, and quality of life deterioration, requiring surgical treatment. ${ }^{4}$ When the symptoms of KOA are not controlled, the replacement of the cartilage for an articular or joint prosthetics is recommended to minimize the incapacities of the arthropathy. Total Knee Arthroplasty (TKA) is just the first step in the process of rehabilitation. Orientations as far as health and therapeutical exercise programs are concerned to recover the muscle strength as well as the movement amplitude (ROM) to control painful and inflammatory symptoms are extremely important to potentialize the results of the orthopedic surgery. ${ }^{5}$

With the increasing prevalence of surgery health systems may become overwhelmed with the demands and costs of rehabilitation, leading to economic and social pressures to reduce intervention time and waiting lines. Hence, new outpatient care models and home exercise guidance are emerging, but it is essential to determine the efficacy, safety and effectiveness of exercises without a therapist's supervision. ${ }^{6}$

Evidences show that in-home therapeutic exercises without professional supervision related to patients submitted to different knee surgeries present positive clinical results and can provide cost reduction. ${ }^{7}$ But the majority of patients with TKA are elderly people and need physiotherapy support during the rehabilitation process. ${ }^{8} \mathrm{~A}$ systematic review evaluated the efficiency of physiotherapy exercises after TKA. Despite the small or few samples of the studies included, good physical and functional outcomes were established in the shortterm. ${ }^{9}$ Determining which model of treatment is effective in the recovery of patients with TKA can contribute to clinical guidelines and health policies. The objective of the systematic review is to analyze randomized clinical trials that tackle different models of rehabilitation 
and compare the effectiveness of the supervised treatment as well as the in-home exercise programs without supervision in patients submitted to TKA.

\section{Methods/design}

\section{Search strategy and selection criteria}

A systematic search was performed in the electronic databases MEDLINE via PubMed, Embase via Elsevier, Lilacs, Trip Database, and Cochrane Library for randomized clinical trials that compared

Table I Characteristics of search strategies in bibliographic databases supervised and unsupervised physical therapy interventions in TKA patients. The search terms were Arthroplasty, Replacement, Knee OR Total Knee Replacements AND Physical Therapy Modalities OR Physical Therapy Techniques AND randomized controlled trial OR controlled clinical trial. The pertinent clinical trials were selected regardless of language or publication medium. The search strategy is presented in Table 1. The study was approved by the Ethics Committee of the institution (CEP0422/2015) and was registered (crd.york.ac.uk/ PROSPERO-CRD:42016050226).

\section{Medline via pubmed}

\#I"Arthroplasty, Replacement, Knee"[Mesh] OR (Arthroplasties, Replacement, Knee) OR(Arthroplasty, Knee Replacement) OR (Knee Replacement Arthroplasties) OR (Knee Replacement, Total) OR (Replacement Arthroplasties, Knee) OR (Replacement Arthroplasty, Knee) OR (Replacement, Total Knee) OR (Knee Replacements, Total) OR (Replacements, Total Knee) OR (Total Knee Replacements) OR (Total Knee Replacement) OR (Arthroplasties, Knee Replacement) OR (Knee Replacement Arthroplasty)

\#2 "Physical Therapy Modalities"[Mesh] OR (Modalities, Physical Therapy) OR (Modality, Physical Therapy) OR (Physical Therapy Modality) OR (Physiotherapy (Techniques)) OR (Physiotherapies (Techniques)) OR (Physical Therapy Techniques) OR (Physical Therapy Technique) OR (Techniques, Physical Therapy)

\#3 ((randomized controlled trial [pt]) OR (controlled clinical trial [pt]) OR (randomized [tiab]) OR (placebo [tiab]) OR (drug therapy [sh]) OR (randomly [tiab]) OR (trial [tiab]) OR (groups [tiab])) AND (humans [mh])

\section{\#4 I,2 \& 3}

\section{Lilacs - literatura latino america e do caribe}

\#I mh:"Artroplastia do Joelho" OR (Substituição Total do Joelho) OR (Artroplastia de Reemplazo de Rodilla) OR (Arthroplasty Replacement Knee) OR MH:E04.555.II0.1 I0.1I5\$ OR MH:E04.650.110.115\$

\#2 MH:"Modalidades de Fisioterapia" OR (Fisioterapia (Técnicas)) OR (Técnicas Fisioterápicas) OR (Modalidade\$ de Fisioterapia) OR (Physical Therapy Modalities)

\section{\#3 \#I and \#2}

\section{Trip database}

(Arthroplasty replacement, Knee) \& (Physical therapy modalities)

\section{EMBASE}

\#I 'knee arthroplasty'/exp OR (arthroplasty, replacement, knee) OR (arthroplasty, knee) OR (knee arthroplasties) OR (knee joint replacement) OR (knee joint replacements) OR (knee reconstruction) OR (knee replacement) OR (knee replacements) OR (reconstruction, knee)

\#2 'physiotherapy'/exp OR (physical therapy) OR (physical therapy (specialty)) OR (physical therapy modalities) OR (physical therapy service) OR (physical therapy specialty) OR (physical therapy techniques) OR (physical treatment) OR (physio therapy) OR (physiotherapy department) OR (therapy, physical)

\#3 random* OR factorial* OR crossover* OR cross AND over* OR placebo* OR 'placebo'/exp OR doubl*AND blind* OR singl* AND blind* OR assign* AND allocate* OR volunteer* OR 'volunteer'/exp OR crossover AND procedure OR double AND 'blind'/exp AND procedure OR randomized AND controlled AND trial OR single AND 'blind'/exp AND procedure OR (controlled AND clinical AND trial) OR (clinical AND trial) AND [embase]/lim

\section{\#4 \#I \#2 \& \#3}

\section{Eligibility criteria}

The inclusion criteria were:

i. Study design: randomized clinical trials (RCTs) (RCTs) (Level I evidence).

ii. Population: males and females of any age submitted to TKA.

iii. Interventions: all physical therapy interventions that compared supervised and unsupervised care.

iv. Exclusion: studies that used the internet for voice communication or videoconferencing.
After excluding the duplicates, two reviewers independently assessed the titles and abstracts of all clinical trials returned by the search, taking down the study characteristics (participants, interventions, comparisons, and outcomes). The pertinent studies were fully read and marked as included or excluded. If the two reviewers disagreed a third researcher was consulted.

\section{Types of outcomes}

The primary outcomes were related to physical and functional aspects: muscle strength, physical function, ROM and atrophy. The secondary outcomes were joint pains and quality of life. 


\section{Risk of bias assessment}

The criteria established by the Cochrane Handbook for Systematic Reviews of Interventions, which measure risk of bias, were used for assessing the methodological quality of the reviewed studies. The assessed items were random sequence generation, allocation concealment, blinding of participants and personnel, incomplete outcome data, and selective reporting, among others. The methods are classified by risk of bias, which can be low (the established criteria were met, consolidating the results); high (a plausible bias is present, reducing result reliability); or unclear (indicates either lack of information or uncertainty over the potential for bias). ${ }^{10}$

\section{Data extraction and statistics}

The reviewed data were tabulated for analysis and structured summarization, and analyzed by the software Review Manager (Revman, version 5.3, Cochrane Collaboration), which calculated the difference between the means of the continuous outcomes and the $95 \%$ confidence interval $(95 \% \mathrm{CI})$ for each estimated point. The patient was the unit of analysis. The statistical heterogeneity of the studies was determined by calculating the statistic $\mathrm{I}^{10}$ and using the chi-square test. When the statistic I 2 exceeded $50 \%$ and the p-value of the chi-square test was equal to or below 0.10 , the studies were considered significantly heterogeneous. The random effects model was used for the meta-analysis of heterogeneous studies. The fixed effects model was used for analyzing studies that were not significantly heterogeneous. The studies were analyzed by subgroups based on follow-up occasion:
i. Two weeks
ii. Six weeks
iii. Three months
iv. Six months
v. One year

\section{Results}

\section{Study characteristics}

Figure 1 shows a flow chart of the study selection process. A total of 1133 studies were found. The Embase database returned the highest number of studies, 586, followed by the PubMed database (230 studies). The Lilacs, Cochrane Library, and Trip databases returned 317 studies. After the exclusion of 121 duplicates, the titles and abstracts of 1012 studies were analyzed for eligibility, regardless of language or publication medium, resulting in the exclusion of another 976 studies. (Thus, 36 studies would be fully read. In this phase only studies with the interventions (supervised $\mathrm{x}$ unsupervised) and population (only TKA patients) of interest remained, numbering five). Table 2 describes the characteristics of the five studies selected for the review of which three were from North America ${ }^{11-13}$ one was from Europe ${ }^{14}$ and one was from Oceania. ${ }^{6}$ The sample sizes varied from 70 to 240 individuals. Females prevailed in all studies, mostly aged 55 to 70 years.

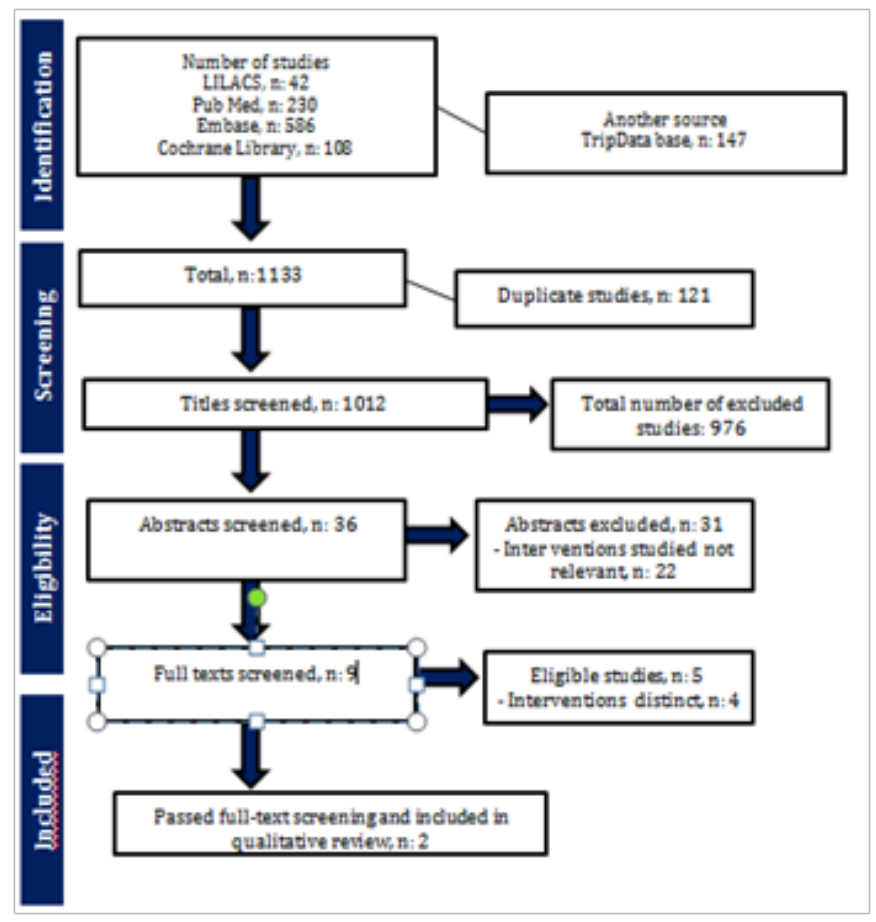

Figure I Flow chart of the study selection process.

Table 2 Characteristics of included studies

\begin{tabular}{|c|c|c|c|c|c|}
\hline Studies & Population & Supervised group & Unsupervised group & Outcomes & Results \\
\hline $\begin{array}{l}\text { Worland } \\
\text { et al. }{ }^{22}\end{array}$ & $\begin{array}{l}80 \text { patients with a } \\
\text { mean age of } 70.2 \\
\text { years submitted to } \\
\text { TKA, } 53 \text { women } \\
\text { and } 27 \text { men }\end{array}$ & $\begin{array}{l}3 \text { times a week- } 2 \text { weeks, } \\
\text { therapeutic exercises- } \\
\text { lower limb strengthening, } \\
\text { gait training and stretching }\end{array}$ & $\begin{array}{l}3 \text { hours a day- } 10 \text { days, } \\
\text { use CPM }\end{array}$ & $\begin{array}{l}\text { ROM: knee flexion and } \\
\text { extension. }\end{array}$ & $\begin{array}{l}\text { On two study occasions ( } 6 \\
\text { weeks and } 6 \text { months) knee } \\
\text { flexion did not differ between } \\
\text { the groups. }\end{array}$ \\
\hline $\begin{array}{l}\text { Kramer et } \\
\text { al. }{ }^{15}\end{array}$ & $\begin{array}{l}\text { I } 60 \text { patients with a } \\
\text { mean age of } 68 \pm 8 \\
\text { years submitted to } \\
\text { TKA, } 9 \text { I women, } \\
26 \text { losses }\end{array}$ & $\begin{array}{l}\text { Therapeutic exercises } \\
\text { performed in group-two } \\
\text { one-hour sessions per } \\
\text { week for } 12 \text { weeks }\end{array}$ & $\begin{array}{l}\text { Exercises for } 12 \text { weeks } \\
\text { and a book with } \\
\text { educational information. } \\
\text { Follow-up by telephone. }\end{array}$ & $\begin{array}{l}\text { ROM: knee flexion; } \\
\text { Function:WOMAC, Knee } \\
\text { Rating Scale, 6MWD, } \\
\text { Sit down and stand up; } \\
\text { Quality of life:SF-36 }\end{array}$ & $\begin{array}{l}\text { The groups showed } \\
\text { improvements at } 12 \text { weeks, } \\
\text { but the difference between the } \\
\text { attendance modalities was not } \\
\text { significant }\end{array}$ \\
\hline
\end{tabular}


Table Continued....

\begin{tabular}{|c|c|c|c|c|c|}
\hline Studies & Population & Supervised group & Unsupervised group & Outcomes & Results \\
\hline $\begin{array}{l}\text { Rajan et } \\
\text { al. }{ }^{23}\end{array}$ & $\begin{array}{l}\text { II } 6 \text { postoperative } \\
\text { TKA patients aged } \\
55-90 \text { years, } 73 \\
\text { women and } 43 \\
\text { men }\end{array}$ & $\begin{array}{l}\text { Outpatient therapeutic } \\
\text { exercises on average 4-6 } \\
\text { times a week-Patients } \\
\text { were assessed on three } \\
\text { occasions ( } 3 \text { months, } 6 \\
\text { months, and I year) }\end{array}$ & $\begin{array}{l}\text { Therapeutic exercises } \\
\text { at home-Patients were } \\
\text { assessed on three } \\
\text { occasions ( } 3 \text { months, } 6 \\
\text { months, and I year) }\end{array}$ & ROM:knee flexion & $\begin{array}{l}\text { The ROM of the two groups } \\
\text { on three occasions did not } \\
\text { differ significantly. The biggest } \\
\text { difference occurred at six } \\
\text { months, when the adjusted } \\
\text { mean difference between } \\
\text { groups was } 2.82 \text { ( } 95 \% \mathrm{Cl}:-0.19 \text {, } \\
5.83 \text {, p }=0.07) \text {; the supervised } \\
\text { group improved slightly but } \\
\text { not significantly }\end{array}$ \\
\hline $\begin{array}{l}\text { Levine et } \\
\text { al. } 12\end{array}$ & $\begin{array}{l}70 \text { patients with a } \\
\text { mean age of } 66.1 \\
\text { years submitted to } \\
\text { TKA }\end{array}$ & $\begin{array}{l}\text { Therapeutic exercises- } \\
\text { Muscle strengthening, } \\
\text { ROM gain, gait training- } 6 \\
\text { months. } 14 \text { days } \\
\text { preoperatively and } 60 \text { days } \\
\text { postoperatively. }\end{array}$ & $\begin{array}{l}\text { NMES and a booklet } \\
\text { with exercises-six } \\
\text { months }\end{array}$ & $\begin{array}{l}\text { ROM: knee flexion and } \\
\text { extension; Function: } \\
\text { WOMAC, KSS and Get } \\
\text { Up-and-Go test }\end{array}$ & $\begin{array}{l}\text { No statistical difference was } \\
\text { found at } 6 \text { weeks or } 6 \text { months } \\
\text { between the groups for knee } \\
\text { ROM, KSS pain, and function, } \\
\text { WOMAC and Get Up-and-go } \\
\text { test. }\end{array}$ \\
\hline Ko et al. ${ }^{6}$ & $\begin{array}{l}233 \text { individuals } \\
\text { with a mean age of } \\
67.3 \text { years }\end{array}$ & $\begin{array}{l}\text { One-to-one/ group- } \\
\text { based therapy: Manual } \\
\text { therapy, cryo therapy, } \\
\text { neuromuscular stimulation, } \\
\text { bracing prescription, } \\
\text { strength training, sensory } \\
\text { motor training, and aerobic } \\
\text { activities-I year }\end{array}$ & $\begin{array}{l}\text { Therapeutic exercise } \\
\text { at home, instructions } \\
\text { provided by DVD and } \\
\text { educational books- } \\
\text { followed for I year }\end{array}$ & $\begin{array}{l}\text { ROM: knee flexion and } \\
\text { extension; Function: } \\
\text { OKS,WOMAC, 6MWD, } \\
\text { Quadriceps Lag (extensor } \\
\text { lag);Quality of life } \\
\text { (Mental and Physical): } \\
\text { SF-I2;Pain:WOMAC; } \\
\text { Therapeutic Satisfaction- } \\
\text { visualanalog scale }\end{array}$ & $\begin{array}{l}\text { The three groups (one-to- } \\
\text { one, group-based therapy, and } \\
\text { monitored home program) did } \\
\text { not present short- and long- } \\
\text { term differences in any of the } \\
\text { study outcomes. }\end{array}$ \\
\hline
\end{tabular}

CPM, continuous passive motion; NMES, neuromuscular electrical stimulation; OKS, oxford knee score; ROM, range of motion; TKA, total knee arthroplasty; WOMAC, western ontario and mcmaster universities; KSS, knee society score; 6MWD, six-minute walk distance

The data of interest included supervised and unsupervised physical therapy sessions conducted at a clinic or at home. The instructions for the unsupervised interventions were provided by e-mails, exercise brochures, and lessons. Four studies compared supervised and unsupervised interventions (two groups). ${ }^{11-14}$ Only one study investigated three physical therapy modalities: supervised individual therapy, supervised group therapy and unsupervised home therapy. ${ }^{6}$

Two studies compared supervised outpatient care with unsupervised home care ${ }^{12,14}$ One study compared the use of continuous passive motion (CPM) devices by an unsupervised group and a supervised group..$^{13}$ Another study in the same line of research compared the use of neuromuscular electrical stimulation (NMES) by an unsupervised group and a supervised group. ${ }^{12}$ One study did not detail the intervention. ${ }^{14}$ Four studies established a relationship between the techniques, namely strength training, encouragement to increase ROM, gait exercises, and joint mobilization and stretching. ${ }^{6,11-13}$ However, none of the studies described the exercises performed during the intervention or their intensity.

\section{Study quality assessment}

(Figures 2) (Figure 3) show the results of the internal quality assessment. Only two studies detailed the random sequence generation process $^{6,14}$ presenting low risk of bias. The other three studies did not describe the random sequence generation process clearly. ${ }^{11-13}$ Only one study described allocation concealment correctly. ${ }^{6}$ The other studies did not provide a full description ${ }^{11-14}$ resulting in unclear risk of bias. Blinding of participants and personnel determines performance bias. Only one study described this methodological step. ${ }^{6}$ Levine et al. ${ }^{11}$ did not detail this step, presenting unclear risk of bias. Three studies ${ }^{12-14}$ did not mention this item, resulting in high risk of bias. Detection bias is avoided by blinding the outcome examiners. Two studies described this procedure well ${ }^{6,13}$ minimizing the risk of bias. Three studies ${ }^{11,12,14}$ described this procedure superficially, resulting in unclear risk of bias. Attrition bias is analyzed by accounting for the existence of incomplete outcomes. Two studies presented all the outcomes, so they were characterized as having low risk of bias for this item. ${ }^{6,14}$ However, three studies did not clarify the study outcomes, which resulted in high risk of bias. ${ }^{11-13}$ Reporting bias regards selective revealing or suppression of information by subjects. Only one study ${ }^{11}$ cited the outcomes, resulting in low risk of bias for this item. Ko et al. ${ }^{6}$ described this item inconclusively, resulting in uncertain risk of bias. Three studies ${ }^{8,15,16}$ did not describe this item clearly, resulting in high risk of bias. Finally, four studies ${ }^{6,11,13}$ did not seem to have other sources of bias. However, Kramer et al. ${ }^{12}$ changed their study design, resulting in high risk of bias.

\section{Results of the meta-analysis}

The five studies included analyzed ROM as outcomes. ${ }^{6,11-14}$ However, the studies presented heterogeneity in relation to the time of the data collecting. Only one research showed ROM after two weeks of rehabilitation ${ }^{11}$ and it did not differ between groups (SD $0.10,95 \%$ CI-5.02 to 5.22). Two clinical trials that measured ROM in six weeks did not find any difference between the supervised group and the unsupervised one $\mathrm{e}^{11,13}$ (SD 1.17, 95\%CI-2.12 to 4.47). Knee flexion ROM at three months did not differ between the groups (SD $3.0 ; 95 \% \mathrm{CI}-0.04$ to 6.04$))^{12,14} \mathrm{~A}$ meta-analysis ${ }^{12-14}$ of the six-month follow-up found no difference between the groups (SD 1.71, 95\%CI0.30 to 3.72 ). ROM at the one-year follow-up also did not differ between groups (SD 2.00, 95\% CI-0.78 to 4.78 ). ${ }^{17}$ These data are presented in Figure 4. Two studies ${ }^{6,12}$ did not present enough data for inclusion in the meta-analysis. We contacted the author but without success. 


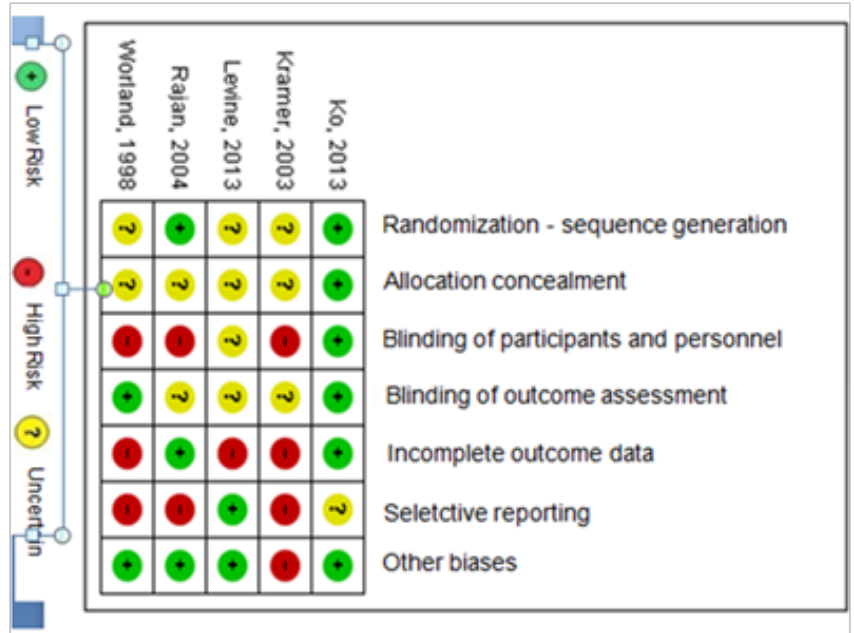

Figure 2 Summary of risk of bias assessment.

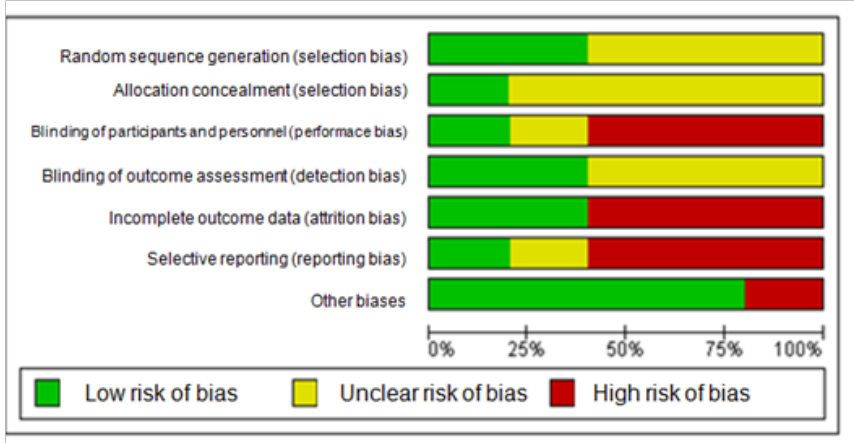

Figure 3 Methodological quality of the reviewed clinical trials expressed as percentages.

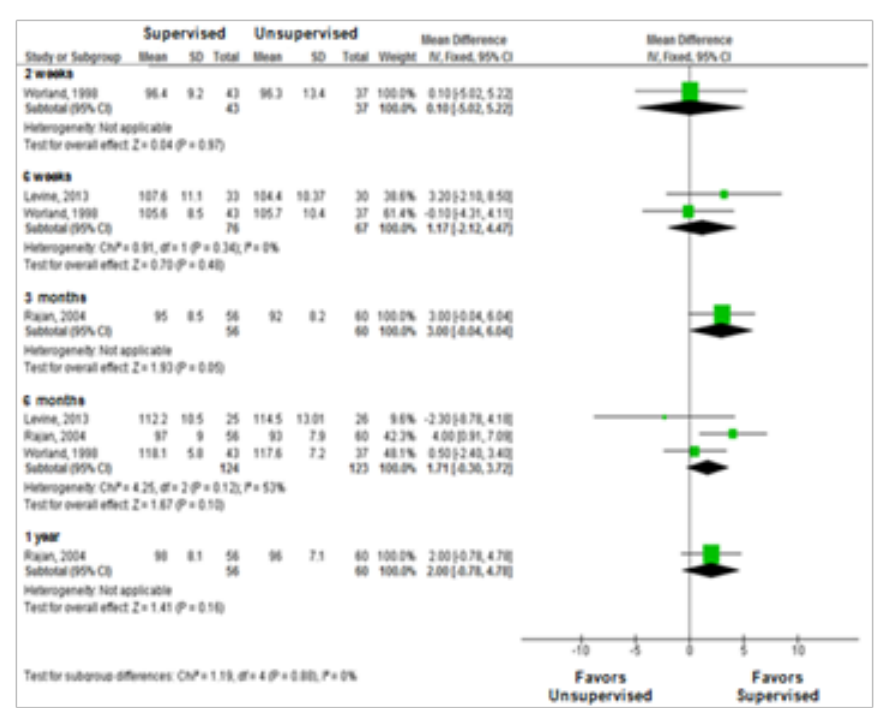

Figure 4 Range of motion (ROM) on different occasions ( 2 weeks, 6 weeks, 3 months, 6 months and I year after total knee arthroplasty) for supervised and unsupervised care.

\section{Discussion}

The present systematic review synthesized randomized clinical trials that compared physical therapy models for TKA patients. The available evidences presented heterogeneity and some studies were limited in the clinical outcomes. Two studies assessed just ROM of patients with TKA. Rojan et al. ${ }^{14}$ analyzed only ROM in the bending of the knees during the rehabilitation process. Functionality, joint pain and quality of life are relevant outcomes in the recovery process for the patients. Three studies made use of WOMAC scale to quantify the functional evolution ${ }^{6,11,15}$ Nevertheless, these three studies differ in the use of functional tests. Kramer et al. ${ }^{12}$ used 6MWD as well as sit down and stand up, Lenine et al. ${ }^{11}$ Applied get up-and-go test and finally Ko et al. ${ }^{6}$ used 6MWD as well as quadriceps lag (extensor lag). Only two randomized clinical trials measured quality of life $\mathrm{e}^{6,12}$ and just one study evaluated that in the long-term. ${ }^{6}$ Quality of life is a very important outcome after TKA, for it is related to functional activities, level of pain, and return to work. ${ }^{18}$ TKA impacts patients' mental and social wellbeing. In a longitudinal study, Linda et al (2015) investigated the functional aspects and quality of life of 851 TKA patients. Patients following knee arthroplasty had better quality of life and aspects related to physical function and pain one year after surgery. To establish functional parameters as well as suitable treatment models is necessary to minimize risks of negative results after TKA. ${ }^{19}$

Some groups question the role of physical therapists in rehabilitation, and their participation in rehabilitation can inconvenience patients and greatly increase treatment cost. ${ }^{20}$ Four studies compared two interventions, one supervised and one unsupervised ${ }^{11-14}$ and one study investigated three models of care, namely supervised individual therapy, supervised group therapy, and unsupervised in-home therapy. ${ }^{6}$ One study used neuromuscular electrical stimulation on the unsupervised group. ${ }^{8}$ Another study used a continuous passive motion (CPM) machine in place of supervised physical therapy to improve ROM. ${ }^{22}$ Both studies did not find differences between the groups.

Majima et al..$^{21}$ who reported a mean knee flexion ROM of $126^{\circ}$ ( \pm 6.5 degrees) after a twelve-week rehabilitation program. Improving knee flexion and extension ROMs is important to improve physical mobility and avoid joint limitations. Supervised rehabilitation protocols allow professionals to follow the patients' progress. The five reviewed studies did not assess muscle strength and atrophy. Recently, a study found that low muscle strength was predictive of TKA in women. A prospective study found that patients following knee surgery had less quadriceps muscle strength and lower physical performance (Timed Up and Go, and Stair Climb Test) than nonsurgery patients.

Among other factors, low muscle strength, especially quadriceps muscle strength, can limit function after TKA. Patients lose as much as fifty percent of their quadriceps muscle strength in the first month after surgery. ${ }^{21}$ For these reasons protocols that assess muscle quality may provide valuable information for the development of effective and safe care modalities. The supervised interventions conducted by the reviewed studies consisted of the following activities: strength training, stretching, sensory motor stimulation, and ROM training. However, these studies did not describe the volume or intensity of the physical therapy exercises performed in the different rehabilitation phases only intervention duration, which varied from six months to one year.

Therefore, future studies in this line of research should include thorough descriptions of the clinical trial interventions, making sure that they are accessible to the readers. The unsupervised interventions provided by two studies consisted of educational instructions by telephone, illustrated books, and DVD videos. ${ }^{6,12}$ In 
developed countries unsupervised interventions or telerehabilitation can help to reduce health care costs and provide good rehabilitation outcomes. ${ }^{22,23}$ However, in underdeveloped countries we can have difficulty in the insertion of this program. The ages of the patients in the reviewed studies varied from 55 to 90 years. This population had compromised functional, cognitive, and social aspects. ${ }^{24-26}$ In order to assess the effect of interventions following TKA these factors must be included as clinical outcomes. However, the reviewed studies did not describe some important outcomes thoroughly. The domain that showed the highest prevalence of uncertainty bias was the allocation concealment. ${ }^{11-14}$ For this domain to satisfy the criteria it is important to describe in detail the method used to conceal the random sequence, using sequentially numbered, opaque, sealed envelopes, and central randomization by a third party to carry out allocation avoiding the acknowledgement of interventions before or during recruitment of individuals.

Among the five eligible studies for the review, only one presented the correct allocation criteria. ${ }^{6}$ Jadad et al. (1996) demonstrated the importance of double-blinding to ensure accurate measurements of effectiveness of a particular intervention. We know the difficulty in blinding the participants to physical therapy interventions; however studies have not shown the blinding of those involved in the research which can interfere with the results. ${ }^{12-14}$ Showed insufficient and inconclusive outcome data, increasing the bias on the findings of the studies. In general, the included studies had methodological limitations. According to the criteria suggested by the Cochrane Collaboration, the presence of bias in methodology weakens and trends the results. ${ }^{10,27-45}$

\section{Conclusion}

The analyzed results presented similarity among the treatment models. But flaws in the available evidences weaken the reliability of the results. In general, this systematic review found methodological flaws and clinical limitations to indicate in-home models without supervision to patients having TKA. Relevant clinical researches with rigorous nmethodological control, and clear descriptions about the physiotherapy conduct are necessary to clarify the benefits of the use of models without supervision to patients submitted to orthopedic surgeries.

\section{Acknowledgements}

None.

\section{Conflict of interest}

The authors declare that they have no competing interests.

\section{References}

1. Stoppiello LA, Mapp PI, Wilson D, et al. Structural associations of symptomatic knee osteoarthritis. Arthritis Rheumatol. 2014;66(11):30183027.

2. Loeser RF, Goldring SR, Scanzello CR, et al. Osteoarthritis: a disease of the joint as an organ. Arthritis Rheum. 2012;64(6):1697-1707.

3. Losina E, Thornhill TS, Rome BN, et al. The dramatic increase in total knee replacementutilization rates in the United States cannot be fully explained by growth in population size and the obesity epidemic. $J$ Bone Joint Surg Am. 2012;94(3):201-207.

4. Bade MJ, Kohrt WM, Stevens LJE. Outcomes before and after total knee arthroplasty compared to healthy adults. J Orthop Sports Phys Ther. 2010;40(9):559-567.
5. Stevens LJE, Bade MJ, Shulman BC, et al. Minimally invasive total knee arthroplasty improves early knee strength but not functional performance: a randomized controlled trial. J Arthroplasty. 2012;27(10):1812-1819.

6. Ko V, Naylor J, Harris I, Crosbie J, et al. One-to-one therapy is not superior to group or home-based therapy after total knee arthroplasty. $J$ Bone Joint Surg Am. 2013;95(21):1942-1949.

7. Wheatley WB, Krome J, Martin DF. Rehabilitation programmes following arthroscopic meniscectomy in athletes. Sports Med. 1996;21(6):447-456.

8. Pellegrini CA, Ledford G, Chang RW, et al. Understanding barriers and facilitators to healthy eating and physical activity from patients either before and after knee arthroplasty. Disabil Rehabil. 2017;1-7.

9. Artz N, Elvers KT, Lowe CM, et al. Effectiveness of physiotherapy exercise following total knee replacement: systematic review and metaanalysis. BMC Musculoskelet Disord. 2015;16(1):15.

10. Jessep SA, Walsh NE, Ratcliffe J, et al. Long-term clinical benefits and costs of an integrated rehabilitation programme compared with outpatient physiotherapy for chronic knee pain. Physiotherapy. 2009;95(2):94-104.

11. Levine M, McElroy K, Stakich V, et al. Comparing conventional physical therapy rehabilitation with neuromuscular electrical stimulation after TKA. Othopedics. 2013;36(3):319-324.

12. Kramer JF, Speechley M, Bourne R, et al. Comparison of clinic and home based rehabilitationn programs after total knee arthroplasty. Clinical Orthop Relat Res. 2003;(410):225-234.

13. Worland RL, Arredondo J, Angles F, et al. Home continuos passive motion machine versus professional physical therapy folloeing total knee replacement. J Arthoplasty. 1998;13(7):784-787.

14. Rajan RA, Pack Y, Jackson H, et al. No need for outpatient physiotherapy following total knee arthroplasty: a randomized trial of 120 patients. Acto Orthop Scand. 2004;75(1):71-73.

15. Piva SR, Teixeira PEP, Almeida GJM, et al. Contribution of hip abductor strength to physical function in patients with total knee arthroplasty. Phys Ther. 2011;91(2):225-233.

16. Ravi B, Croxford R, Reichmann WM, et al. The changing demographics of total joint arthroplasty recipients in the United States and Ontario from 2001 to 2007. Best Pract Res Clin Rheumatol. 2012;26(5):637-647.

17. Lange AK, Vanwanseele B, Fiatarone SMA. Strength training for treatment of osteoarthritis of the knee: A systematic review. Arthritis Rheum. 2008;59(10):1488-1494.

18. Pozzi F, Snyder ML, Zeni J. Physical exercise after knee arthroplasty: a systematic review of controlled trials. Eur J Phys Rehabil Med. 2013;49(6):877-892.

19. Restogi R, Chesworth BM, Davis AM. Change in patient concerns following totalknee arthroplasty described with the international classification of functioning, disability and health: a repeated measures design. Health Qual Life Outcomes. 2008;(11)6:112-120.

20. Miyagawa H, Ikemoto T, Honjo H, Akao M, et al, One-year results of voluntary-based supervised exercise or treatment at orthopedic clinic for radiographic severe knee osteoarthritis. J Phys Ther Sci. 2016;28(3):906910 .

21. Majima T, Nishike O, Sawaguchi N, et al. Patella eversion reduces early knee range of motion and muscle torque recovery after total knee arthroplasty: comparison between minimally invasive total knee arthroplasty and convention total knee arthroplasty. Arthritis. $2011 ;(12): 1-5$

22. Kirk SNJ, McGough EL. Physical exercise and cognitive performance in the elderly: current perspectives. Clin Interv Aging. 2014;9(18):51-62. 
23. Landry MD, Jaglal SB, Wodchis WP, et al. Rehabilitation services after total joint replacement in Ontario, Canada: can 'prehabilitation' programs mediate an increasing demand? Int J Rehabil Res. 2007;30(4):297-303.

24. Roos, EM, Juhl CB. Osteoarthritis 2012 year in review: rehabilitation and outcomes. Osteoarthritis Cartilage. 2012;20(12):1477-1483.

25. Higgins JP, Altman DG, Gotzsche PC, et al. The Cochrane Collaboration's tool for assessing risk of bias in randomized. BMJ. 2011;343(18):1-9.

26. Stevens LJE, Balter JE, Kohrt WM, et al. Quadriceps and hamstrings muscle dysfunction after total knee arthroplasty. Clin Orthop Relat Res. 2010;468(9):2460-2468

27. Labraca NS, Castro SAM, Matarán PGA, et al. Benefits of starting rehabilitation within 24 hours of primary total knee arthroplasty: randomized clinical trial. Clin Rehabil. 2013;25(6):557-566.

28. Arnold JB, Mackintosh S, Olds TS, et al. Improvements in knee biomechanics during walking are associated with increased physical activity after total knee arthroplasty. J Orthop Res. 2015;33(12):18181825 .

29. Imoto AM, Peccin MS, Trevisani VFM. Exercícios de fortalecimento de quadríceps são efetivos na melhora da dor, função e qualidade de vida de pacientes com osteoartrite do joelho. Acta Ortopédica Brasileira. 2012;20(3):174-179.

30. Maruyama T, Sawada Y, Kubo S, et al. Postoperative changes in knee joint function of total knee arthroplasty patients. J Phys Ther Sci. 2011;23(5):719-724.

31. Mahomed NN, Davis AM, Hawker G, et al, Wright JG. Inpatient compared with home-based rehabilitation following primary unilateral total hip or knee replacement: a randomized controlled trial. J Bone Joint Surg Am. 2008;90(8):1673-1680.

32. Haynes RB, Ackloo $\mathrm{E}$, Sahota $\mathrm{N}$, et al. Interventions for enhancing medication adherence. Cochrane database syst revi. 2008;16(2):CD000011

33. Coppola SM, Collins SM. Is physical therapy more beneficial than unsupervised home exercise in treatment of post surgical knee disorders? a systematic review. Knee. 2009;16(3):171-175.
34. Hailey D, Roine R, Ohinmaa A, et al. Evidence of benefit fromtelerehabilitation in routine care: a systematic review. $J$ Telemed Telecare. 2011;17(6):281-287.

35. Chehad EF, Favre J, Erhart HJC, et al. Baseline knee adduction and flexion moments during walking are both associated with five year cartilage changes in patients with medial knee osteoarthritis. Ostearthritis Cartilage. 2014;22(11):1833-1839.

36. Silva RR, Santos AA, Sampaio CJJ, et al. Quality of life after knee arthroplasty: systematic review. Rev Bras Ortop. 2014;19(5):520-527.

37. Bhende H, Laud N, Deore S, et al. Total knee arthroplasty in vascular malformation. Indian J Orthop. 2015;49(5):569-572.

38. Mizner RL, Petterson SC, Snyder ML. Quadriceps strength and the time course of functional recovery after total knee arthroplasty. J Orthop Sports Phys Ther. 2005;35(7):424-436.

39. Fernandez A, Sturmberg J, Lukersmith S, et al. Evidence-based medicine: is it a bridge too far? Health Res Policy Syst. 2015;6(13):1-9.

40. Kluzek S, Newton JL, Arden NK. Is osteoarthritis a metabolic disorder? Br Med Bull. 2015;115(1):111-121.

41. Hillcoat NS. The meaning of "independence" for older people indifferent residential settings. J Gerontol B Psychol Sci Soci. 2014;69(3):419-430.

42. Harmelink KEM, Zeegers AVCM, Hullegie W, et al. Are There Prognostic Factors for One-Year outcome after total knee arthroplasty? A systematic review. J Arthroplasty. 2017;32(12):3840-3853.

43. Zeni JA Jr, Axe MJ, Snyder ML. Clinical predictors of elective total persons with end-stage knee osteoarthritis. BMC Musculoskelet Disord. 2010;11(6):1-8.

44. Kim TK, Kwon SK, Kang YG, et al. Functionaldisabilities and satisfaction after total knee arthroplasty in female Asian patients. $J$ Arthroplasty. 2010;25(3):458-464.

45. Mitchell C, Walker J, Walters S, et al. Costs and effectiveness of preand post-operative home physiotherapy for total knee replacement: randomized controlled trial. J Eval Clin Pract. 2005;11(3):283-292. 